\title{
Regional Seismographic Station Management System
}

\author{
Yibing Dong ${ }^{1,2, a}$, Xiang Wang ${ }^{2, b}$, and Yinjie Zhu ${ }^{3, c}$ \\ ${ }^{1}$ University of Science \& Technology of China, China \\ ${ }^{2}$ Seismological Bureau of Hebei Province, China \\ ayuehun06@163.com, bwangx@eq-he.ac.cn, ${ }^{\mathrm{c}} 526252372 @ q q . c o m$
}

\begin{abstract}
Keywords: regional seismographic network; seismographic sensors; management information system; WebGIS; JMS

Abstract. Design and implementation of a system is introduced, which is designed to provide tools for station-management in the regional telemetry seismographic network, with a visual way to process data on management and geographic of seismographic stations. Web services including WebGIS, station-information management, state-of-health $(\mathrm{SOH})$ data monitoring, and station-fault statistics are provided. GeoServer and Openlayers is used to provide WebGIS services to implement the spatial visualization of the seismographic network. ActiveMQ is used to publish the SOH data collected from sensors with unified protocols and interfaces. In practice, the software has been used in the regional seismic network of Hebei Province of China, leading to an enhancement of QOS of the seismic network.
\end{abstract}

\section{Introduction}

The regional seismographic network is a multi-station, evenly distributed, state-of-the-art digital network that provides data and information for earthquake locations, earthquake hazard mitigation, and earthquake emergency response. The network is a distributed system consisting of a center and several stations equipped with information infrastructure including sensors, servers and applications. Its instrumentation measures and records with high fidelity all seismic vibrations possible from high-frequency, strong ground motions near an earthquake to the slowest global Earth oscillations excited by great earthquakes[1].

The key goal of a seismographic station has always been recording high-quality seismic wave forms, which depends on both external and internal factors. The external is related to the environment condition, and the internal depends on instrumentation and management afterwards. In a regional seismographic network, station-management plays a fundamental role, with purpose to enhance QOS of the network. Contents of station-management include maintenance, monitoring and reforming of the stations' infrastructure. During running, it is inevitable that errors occurring leading to an interruption of the real-time wave data transmission. So it's necessary to monitor the state-of-health $(\mathrm{SOH})$ of instrumentation and make statistics and analysis of the faults periodically to master the running characters and support decision-making. These tasks need to be carried out with special-developed and well-designed software.

\section{Requirements}

Seismic Station Maintenance. In the regional seismic network, seismic station maintenance usually contains the following procedures: Step 1. Check the status of the information infrastructure, mainly about the services transmitting wave-form data and instrumentation of stations. Step 2. When meeting with errors, engineers need to find out the incentives and assess the impact as quickly as possible. In practice, there are usually 2 kinds of errors, fault or exception. Fault means fatal mistakes cause an transmission interruption of the real-time wave-form data, and exception means something wrong with the instruments such as certain $\mathrm{SOH}$ value exceeds its normal range e.g. (Table 1). Step 3. According to the previous assessment, take measures to correct the mistakes. Sometimes several 
common errors will end automatically after a moment, and serious errors always need to be solved on the scene.

Table 1. Data Dictionary of SOH.

\begin{tabular}{cccc}
\hline Id & Name & Description & Normal Range \\
\hline 1 & Pwr_input & Input voltage @ V & {$[-9,18]$} \\
2 & Pwr_output & Output voltage @ V & {$[-9,18]$} \\
3 & Pwr_battery & Battery voltage @ V & {$[0,9]$} \\
4 & Dsp_temp & Temperature @ C & {$[-20,60]$} \\
5 & Clk_diff & Clock Error @ $\mu$ S & {$[-100,100]$} \\
6 & Frq_diff & Frequency Error @ PPB & {$[-10,10]$} \\
7 & Drive_free_space & Free Space @ 100\% & {$[20,100]$} \\
8 & Z_mp_volts & Mass Position of Chanel UD @ V & {$[-1,1]$} \\
9 & N_mp_volts & Mass Position of Chanel NS @ V & {$[-1,1]$} \\
10 & E_mp_volts & Mass Position of Chanel EW @ V & {$[-1,1]$} \\
\hline
\end{tabular}

Functional Requirements. The system is designed to provide real-time online services for station-management in the regional seismographic network, with the following key features:WebGIS platform: support spatial visualization of the station distribution and basic geographic data, offering browsing, configuration, selection and measuring tools.Station information management: support management of the logs and archives generated during station-maintenance process, including station-basic-parameters, station-maintenance-logs and instrument-management-logs.SOH monitoring: collect SOH data from stations periodically, turn the data to JMS messages and send them to ActiveMQ. Support threshold configuration and exception alarming. Station fault statistics: support classification statistics on the fault records with report outputting.

\section{Structure Design}

The system uses a distributed multi-layer structure, with 5 main services (Figure 1).

$\mathrm{SOH}$ Access Service, written in java, collect $\mathrm{SOH}$ data from instruments, turn the data into BytesMessage and send them to the message queue named SOH.TOPIC managed by ActiveMQ (AMQ).

AMQ, an open source message broker as well as a full JMS client[2]. Java Message Service (JMS) is a messaging standard that allows application components based on the Java Enterprise Edition (Java EE) to create, send, receive, and read messages. It allows the communication between different components of a distributed application to be loosely coupled, reliable, and asynchronous[3]. AMQ support PostgreSQL(PG) for message persistence. With PostgresqlJDBCAdapter, the SOH message can be stored into PG.

PG/PostGIS. PostGIS is a spatial database extender for PG. It adds support for geographic objects allowing location queries to be run in SQL[4]. In our database, the Business-related data is stored in PG, and geographic data stored in PostGIS.

Apache Tomcat, an Web container[5], the Web Service provider, where integrated all the business logic and WebGIS service, plays the key role of the system . A geographical information system (GIS) is a system designed to capture, store, manipulate, analyze, manage, and present all types of spatial or geographical data[6].

GeoServer is an open source software server written in Java that allows users to share and edit geospatial data. Designed for interoperability, it publishes data from any major spatial data source using Open Geospatial Consortium (OGC) Web Feature Service (WFS) and Web Coverage Service (WCS) standards, as well as a high performance certified compliant Web Map Service (WMS)[7].

Web Front-End, define the user interface, where JSP and OpenLayers adopted. OpenLayers is an open source JavaScript library for displaying map data in web browsers. It provides an API for building rich web-based geographic applications similar to Google Maps and Bing Maps[8]. 


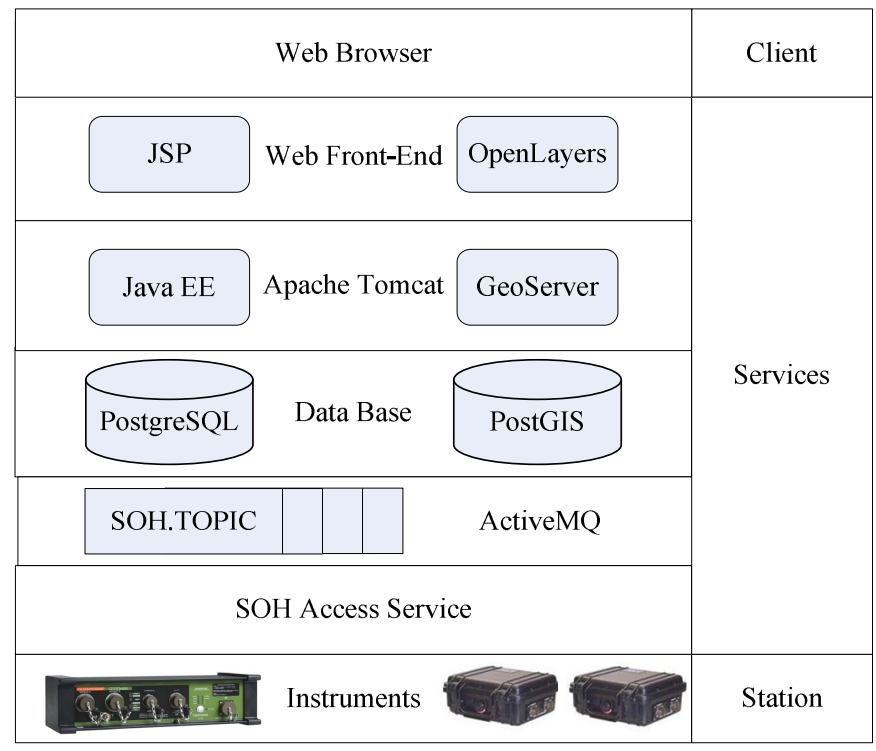

Figure 1. Structure of the System

\section{Front-End Design}

The Main Page. As shown in Figure 2(a), the main page can be divided into 3 parts, the system LOGO and navigation buttons on the top, station E-map in the center, and E-map options on the right. The navigation buttons link to different modules such as station-management, $\mathrm{SOH}$-monitoring and system-settings. The E-map options provide all the optional layers. On the E-map, each triangle stands for a seismographic station, and its color indicates the stations' real-time status, where green means that both the real-time waveform data transmission service and the instrument runs well; red means there's an exception may happened, when the data service runs well, but at least one of the $\mathrm{SOH}$ parameters exceeds its range; and black means there's a fault may happened leading to the data service running timeout. Once a station turn into red or black, it must be especially checked by the engineers.

Station Management. Click the button named station-management will open a pop-up menu from left of the map as shown in Figure 2(b). There are 3 sub-menus from top to bottom including station-basic-parameter-management, instrument-log-management and station-maintenance-log-management. Figure 2(b) shows the sub-menu of station-basic-parameter-management, there is a station list with the stations' basic information shown, which supports sorting and tabbed browsing. The buttons to add, delete, modify and search records are provided on the toolbar of the menu. When a guest enters the system, he will only be allowed to view part of the open data, and only be the administrators privileged to edit all of the data.

SOH Monitoring. In the station list, double-click a station will open its SOH monitoring page as shown in Figure 2(c), which updates contents automatically . Top of the page is a list of the SOH data newly received, and bottom is the dash board synchronized with the list, with hand pointing to the green area meaning well and red area meaning not. Users can monitor the instruments' real-time status via observing the dashboard changes, and also can customize each parameter's threshold range with setting the system configuration.

Station fault statistic. Users can open the page of station-fault-statistics via a hyperlink on the station-management menu as shown in Figure 2(d). Here, users need to fill in the blanks with network code, station code, start time and end time, click the "Statistics" button, and then the system will operate statistics and print result charts on the screen. From the result, users can get the proportion of each type of fault, and find out the most serious faults within a period. For example, Figure 2(d) shows the statistical result of faults in Hebei seismic network at 2014. It can be seen that power supply stopped accounts for the highest proportion, suggesting that engineers should take measures to improve the power supply situation, replacing back-up batteries timely e.g. 


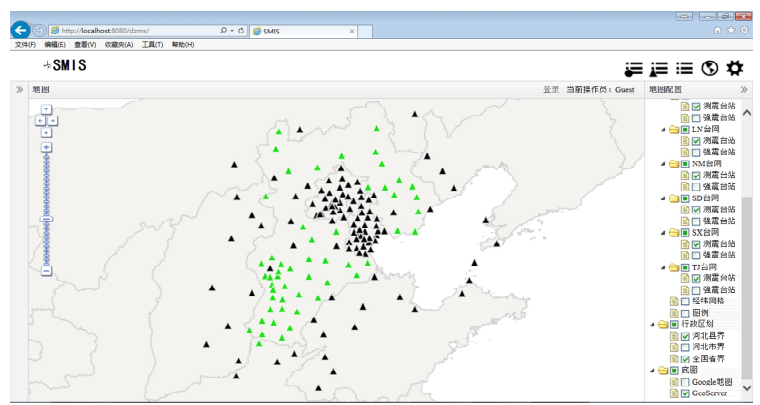

(a)

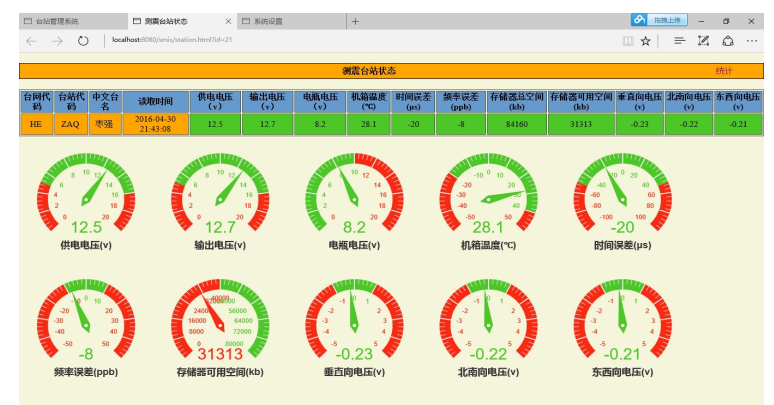

(c)

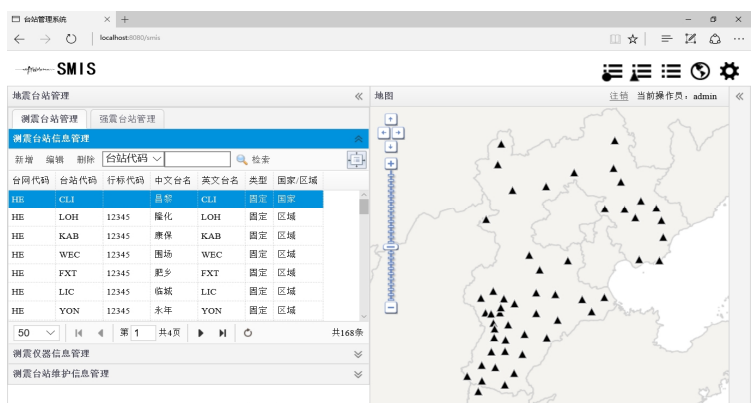

(b)

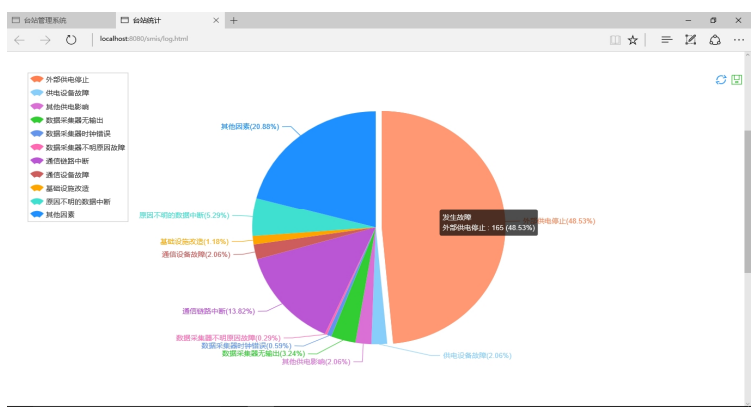

(d)

Figure 2. Some Pages of the System

\section{Conclusions}

A software is designed to provide tools for station-management in the regional telemetry seismographic network, with a visual way to process data on management and geographic of seismographic stations. Web services including WebGIS, station-information management, state-of-health $(\mathrm{SOH})$ data monitoring, and station-fault statistics are provided. In practice, the software has been used in the regional seismic network of Hebei Province of China, leading to an enhancement of QOS of the seismic network..

\section{Acknowledgments}

This work was financially supported by the Science and Technology Support Program of Hebei Province (15275402D).

\section{References}

[1] Information on http://www.iris.edu/hq/programs/gsn.

[2] Information on http://activemq.apache.org/cross-language-clients.html.

[3] Information on http://www.oracle.com/technetwork/java/jms/index.html.

[4] Information on http://www.postgis.net/.

[5] Information on https://tomcat.apache.org/.

[6] Kenneth E. Foote and Margaret Lynch, Geographic Information Systems as an Integrating Technology: Context, Concepts, and Definitions". The Geographer's Craft Project, Department of Geography, The University of Colorado at Boulder. Retrieved 21 Apr 2015.

[7] Information on http://docs.geoserver.org/stable/en/user/introduction/overview.html.

[8] Information on http://openlayers.org/en/latest/doc/tutorials/introduction.html. 